\title{
Article \\ A Screening of the MMV Pandemic Response Box Reveals Epetraborole as A New Potent Inhibitor against Mycobacterium abscessus
}

\author{
Taeho Kim ${ }^{1}$, Bui-Thi-Bich Hanh ${ }^{1}$, Boeun Heo ${ }^{2}$, Nguyenthanh Quang ${ }^{2}$, Yujin Park ${ }^{2}$, Jihyeon Shin ${ }^{2}$, \\ Seunghyeon Jeon ${ }^{3}$, June-Woo Park ${ }^{4,5}$, Kirandeep Samby ${ }^{6}$ and Jichan Jang ${ }^{2, *}$
}

Citation: Kim, T.; Hanh, B.-T.-B.; Heo, B.; Quang, N.; Park, Y.; Shin, J.; Jeon, S.; Park, J.-W.; Samby, K.; Jang, J. A Screening of the MMV Pandemic Response Box Reveals Epetraborole as A New Potent Inhibitor against Mycobacterium abscessus. Int. J. Mol. Sci. 2021, 22, 5936. https://doi.org/ 10.3390/ijms22115936

\section{Academic Editors:}

Natalie Eva Nieuwenhuizen and Joanna Evans

Received: 16 March 2021

Accepted: 27 May 2021

Published: 31 May 2021

Publisher's Note: MDPI stays neutral with regard to jurisdictional claims in published maps and institutional affiliations.

Copyright: (c) 2021 by the authors. Licensee MDPI, Basel, Switzerland. This article is an open access article distributed under the terms and conditions of the Creative Commons Attribution (CC BY) license (https:// creativecommons.org/licenses/by/ $4.0 /$ )
1 Division of Applied Life Science (BK21 Four Program), Research Institute of Life Science, Gyeongsang National University, Jinju 52828, Korea; taeho12349@gmail.com (T.K.); hanhm0515006@gstudent.ctu.edu.vn (B.-T.-B.H.)

2 Molecular Mechanisms of Antibiotics, Division of Life Science, Department of Bio \& Medical Big Data (BK21 Four Program), Research Institute of Life Science, Gyeongsang National University, Jinju 52828, Korea; hbo0113@naver.com (B.H.); nguyenthanhquang1411@gmail.com (N.Q.); syryejr@naver.com (Y.P.); jihyeon2531@naver.com (J.S.)

3 Division of Life Science, Gyeongsang National University, Jinju 52828, Korea; sarahpop@naver.com

4 Department of Environmental Toxicology and Chemistry, Korea Institute of Toxicology, Jinju 52843, Korea; jwpark@kitox.re.kr

5 Human and Environmental Toxicology Program, Korea University of Science and Technology (UST), Daejeon 34113, Korea

6 Medicines for Malaria Venture (MMV), 20, Route de Pré-Bois, 1215 Geneva, Switzerland; sambyk-consultants@mmv.org

* Correspondence: jichanjang@gnu.ac.kr; Tel.: +82-055-772-1368

\begin{abstract}
Mycobacterium abscessus is the one of the most feared bacterial respiratory pathogens in the world. Unfortunately, there are many problems with the current $M$. abscessus therapies available. These problems include misdiagnoses, high drug resistance, poor long-term treatment outcomes, and high costs. Until now, there have only been a few new compounds or drug formulations which are active against $M$. abscessus, and these are present in preclinical and clinical development only. With that in mind, new and more powerful anti-M. abscessus medicines need to be discovered and developed. In this study, we conducted an in vitro-dual screen against M. abscessus rough (R) and smooth (S) variants using a Pandemic Response Box and identified epetraborole as a new effective candidate for M. abscessus therapy. For further validation, epetraborole showed significant activity against the growth of the M. abscessus wild-type strain, three subspecies, drug-resistant strains and clinical isolates in vitro, while also inhibiting the growth of M. abscessus that reside in macrophages without cytotoxicity. Furthermore, the in vivo efficacy of epetraborole in the zebrafish infection model was greater than that of tigecycline. Thus, we concluded that epetraborole is a potential anti-M. abscessus candidate in the M. abscessus drug search.
\end{abstract}

Keywords: Mycobacterium abscessus; epetraborole; benzoxaboroles; drug discovery; antibiotics

\section{Introduction}

Despite great strides in the field of infectious disease over the past century, Mycobacterium abscessus (hereafter referred to as $M a b$ ) complex infection has remained a leading cause of morbidity and mortality in cystic fibrosis (CF) patients [1]. Mab infections are deadly complicated to treat due to their mechanisms for drug resistance and biofilm generation [2]. Although there is no compelling agent for Mab treatment: clarithromycin (CLA), amikacin (AMK), and cefoxitin (CFX) are currently used as a drug combination for 1 to 2 months, followed by an oral maintenance regimen, usually with a fluoroquinolone, based on the recommendations of experts [3]. However, long-term and intensive combination therapy has shown high rates of treatment failure, recurrences, and adverse effects. 
Furthermore, the use of this combination therapy resulted in only a 50\% efficacy rate even with adjunctive surgery and most patients either relapse or die [4]. Therefore, more efficacious drugs are urgently needed.

In this context, high throughput screening has been conducted with Mab using various chemical libraries [5-12]. Unfortunately, there are not many active new drug candidates in either the clinical or discovery phase. This may be due to the extremely low hit rate of chemical drug screens that target Mab [6]. Even selected hits have not been further developed successfully into clinical trials. Fundamentally, mycobacteria contain a very thick cell membrane called mycomembrane which is composed of peptidoglycan, arabinogalactan covalently linked to an inner leaflet of long-chain mycolic acids. Furthermore, Mab also contains a large variety of extractible lipids from the outer leaflet of the mycomembrane, such as glycopeptidolipids (GPLs) [13,14]. Thus, these thick cell envelopes are a major permeability barrier for drug penetration. Besides, $M a b$ is an intracellular pathogen. During infection, Mab is engulfed by human macrophages, overcomes a host's immune defense mechanisms, replicates, and resides inside human immune cells [15]. Therefore, only compounds with a high enough penetration ability to pierce the macrophage plasma membrane and the thick cell membrane of the Mab will lead to an inhibition of intracellular mycobacterial growth. To make matters worse, Mab also contains efflux pump-related genes that can export chemical agents, as well as possessing some enzymes that can modify drugs and targets which makes it difficult for drugs to be functional [16-18].

Here, we screened the Pandemic Response Box against Mab to identify a promising anti-Mab agent. The Pandemic Response Box is a drug library assembled from the Medicines for Malaria Venture (MMV) and Drugs for Neglected Diseases initiative (DNDi), in association with scientists from both industry and academia, to foster new research into treatments for pandemic diseases. This library contains 400 structurally diverse compounds (201 antibacterials, 153 antivirals, and 46 antifungals) for screening against infective and neglected diseases. Using this valuable library, we identified one potent candidate named epetraborole (also known as AN 3365; code number: GSK 2251052) that showed excellent growth inhibitory activity against $M a b$ in the low nanomolar range. We further characterized the hit molecules regarding their bacterial inhibitory effect inside both macrophage and zebrafish models of infection.

\section{Results}

\subsection{Drug Screening against Mab Using a Pandemic Response Box}

For the screen, the library was obtained in plates from the Medicines for Malaria Venture (MMV, Geneva, Switzerland). It was then diluted to $2 \mathrm{mM}$ and transferred directly into assay plates to yield a primary screen concentration of $20 \mu \mathrm{M}$ (final concentration of $2 \%$ DMSO). The primary screen conditions were used for two different strains (Mab subsp. abscessus CIP 104536T S- and R-variants) and 80\% growth inhibition was plotted. The single-point assays produced 3 compounds (primary hit rate $0.75 \%$ ) that had a $>80 \%$ inhibitory effect against both Mab variants in cation-adjusted Mueller-Hinton (CAMH) medium (Figure 1).

The three hits were epetraborole (ETB), eravacycline (ERV), and bedaquiline (BDQ). The 3 active hits were then confirmed by dose-response curve determination to generate inhibitory concentration (IC) by resazurin microtiter assay (REMA) and their half inhibitory concentrations $\left(\mathrm{IC}_{50}\right)$ were below $1 \mu \mathrm{M}$, which is more potent to that of reference compound CLA. The $\mathrm{IC}_{50}$ values were $0.4,0.1$ and $0.8 \mu \mathrm{M}$ for ETB, ERV, and BDQ, respectively (Table 1). Because recent studies have shown the anti-Mab effect of ERV and BDQ, we will describe the findings of only ETB against Mab in this study. 


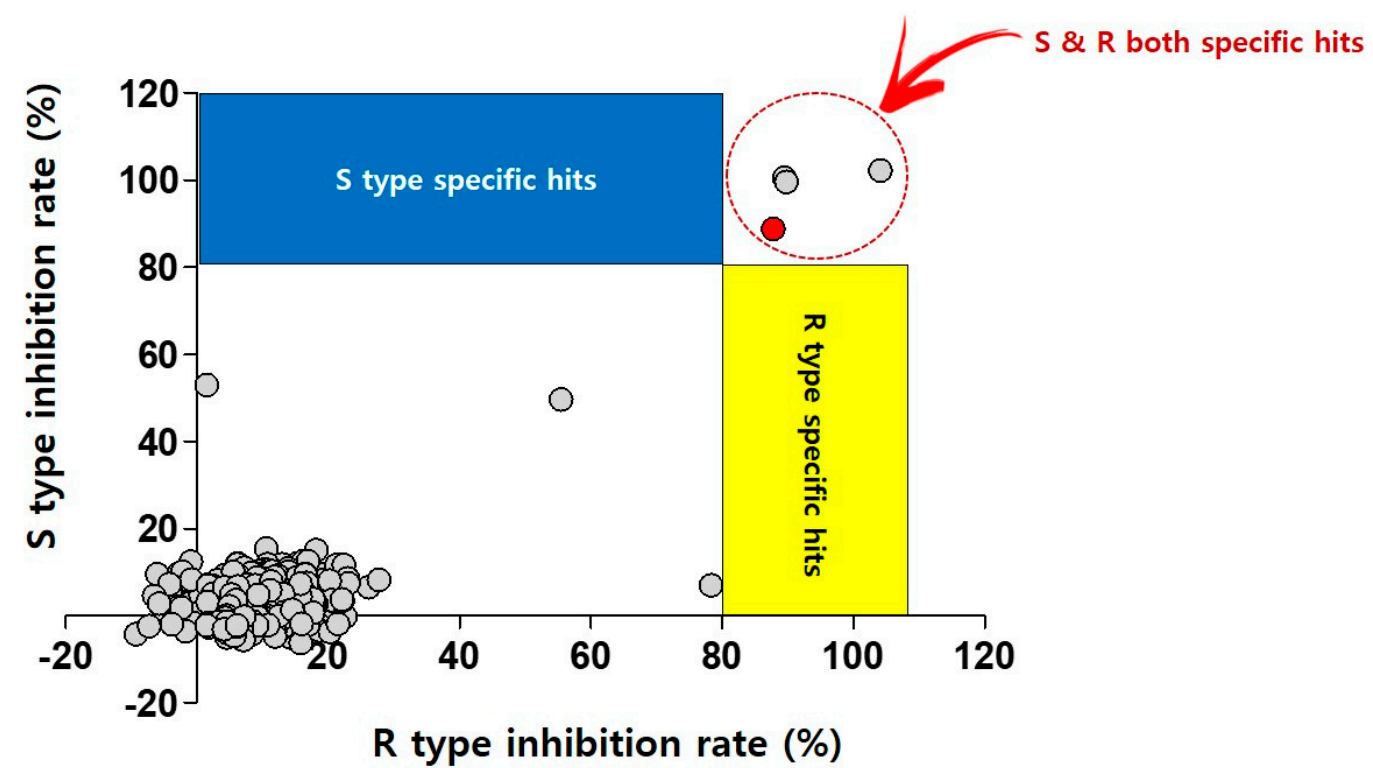

Figure 1. Dual screening of the Pandemic Response Box using Mab S and R variants. Scatter plot distribution showing the results of the Mab S and R variant dual screening of the Pandemic Response Box using resazurin reduction assay. A total of 400 compounds from the Pandemic Response Box were screened at $20 \mu \mathrm{M}$ against both $\mathrm{Mab}$ S and R variants. Growth inhibition of at least $80 \%$ was defined as the cut-off which resulted in 3 hits ( $0.75 \%$ hit rate). Red closed circle indicates CLA as positive control.

Table 1. Chemical structure and half maximal inhibitory concentration $\left(\mathrm{IC}_{50}\right)$ values of the 3 most potent $M a b$ hits.

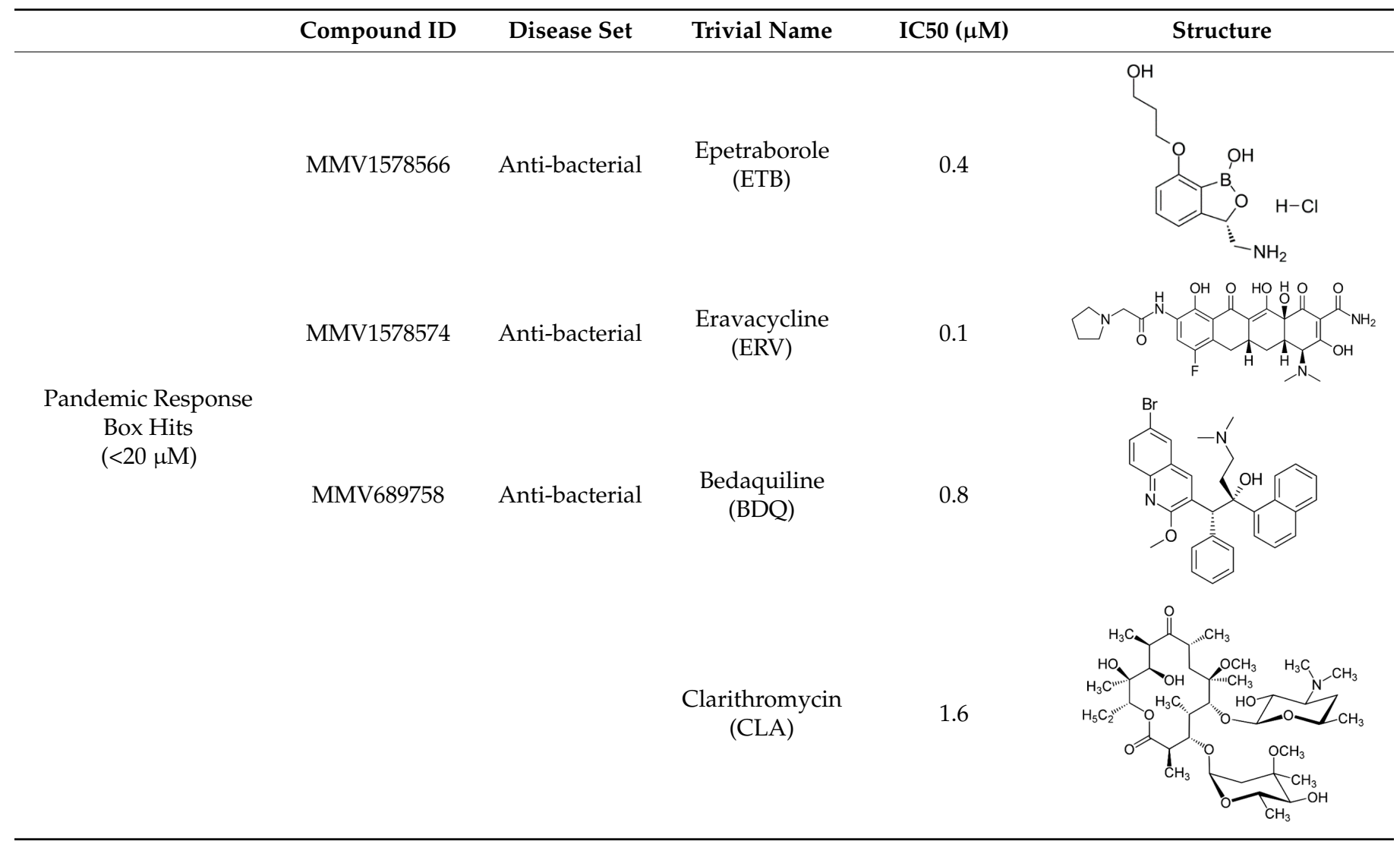




\subsection{ETB Has Mab Killing Effect and Exhibits Potent Activity against Mab Subspecies, Clinical} Isolates, and Drug-Resistant Strains

Time-kill assays were performed using ETB for the Mab-S variant (CIP 104536 ${ }^{\mathrm{T}}$ ). Figure 2 shows the pattern of growth and killing of Mab by ETB at different concentrations. The CLA was used as a negative control. After a short declined lag phase at day 1, an increase in CFU (colony forming unit) was observed at $5 \times$ CLA concentration. Furthermore, it reached the highest CFU counts after 5 days of incubation. In contrast, ETB exhibited bactericidal activity at concentrations of 2.5-, and was 5-fold higher than the $\mathrm{IC}_{50}$ determined by the REMA (Table 1). Only 1 day later, ETB started to decrease the bacterial density with its maximum decrease after 5 days at $2.5 \times$ and $5 \times$ concentrations of $\mathrm{IC}_{50}$. The bacterial killing effect was not observed at $0.5 \times$ MIC of ETB.

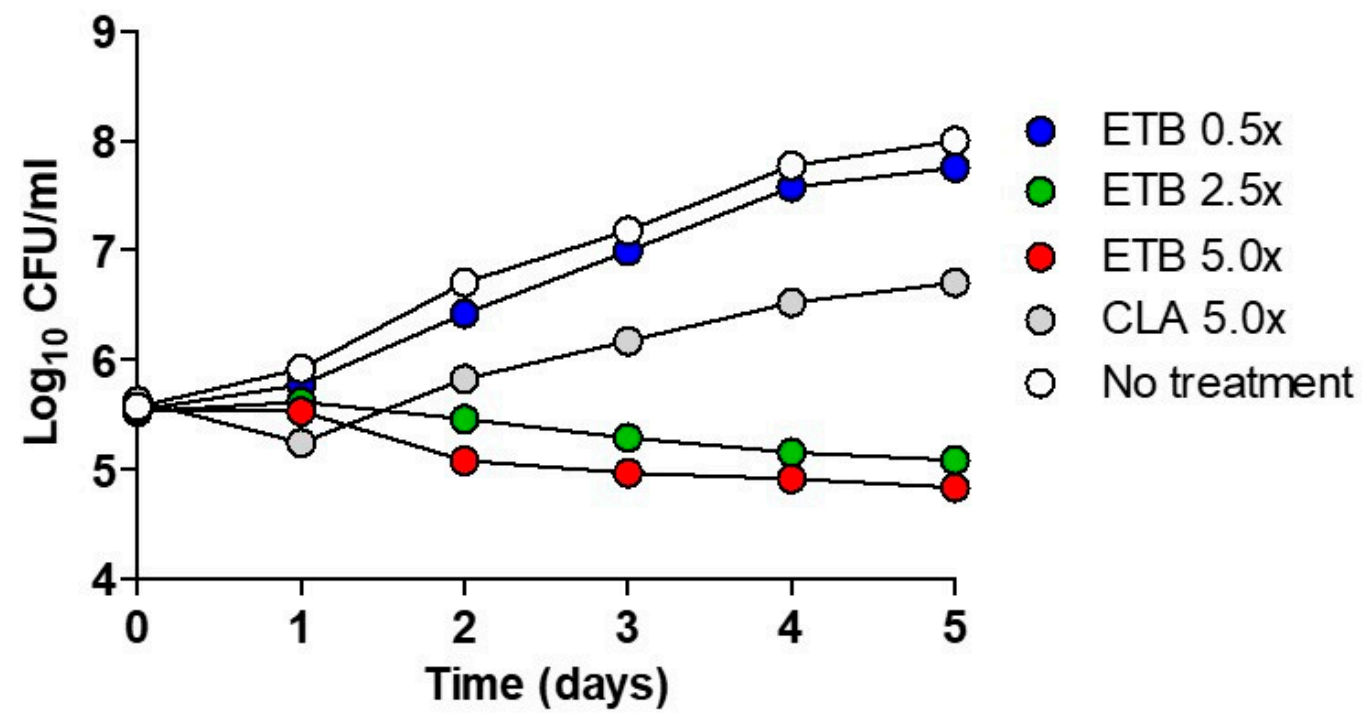

Figure 2. Time-kill curves of ETB against Mab. The bacteria were grown in a liquid culture (Middlebrook 7H9 medium) in the presence of the indicated concentrations of ETB and was plated on a 7H10 Middlebrook agar plate. Antibiotic concentrations are indicated by different symbols. Each point represents the mean of triplicate determinations.

We continued to evaluate the concentrations that caused inhibition of growth of Mab using ETB for three different Mab subspecies comprising Mab subsp. abscessus CIP $104536^{\mathrm{T}}$, Mab subsp. massiliense CIP108297 ${ }^{\mathrm{T}}$, and Mab subsp. bolletii CIP108541 ${ }^{\mathrm{T}}$. As shown in Table 2, a decrease in fluorescence was observed, indicating a dose-dependent inhibitory effect after incubating Mab strains with the ETB for 5 days. All the subspecies tested were susceptible to ETB. Mab subsp. massiliense CIP108297 ${ }^{\mathrm{T}}$ showed the lowest IC value ( $\mathrm{IC}_{50}$ values of $0.11 \mu \mathrm{M}$ and $\mathrm{MIC}_{90}$ values of $0.33 \mu \mathrm{M}$ ) and Mab subsp. abscessus CIP $104536^{\mathrm{T}}$ showed the highest $\mathrm{IC}_{50}$ and $\mathrm{IC}_{90}$ value at $0.25 \mu \mathrm{M}$ and $0.56 \mu \mathrm{M}$ respectively. Meanwhile, Mab subsp. bolletii CIP108541 ${ }^{\mathrm{T}}$ was $0.22 \mu \mathrm{M}$ for $\mathrm{IC}_{50}$.

In addition, we conducted IC determination tests to know whether ETB could inhibit the growth of drug-resistant strains that were laboratory induced at high concentrations of amikacin (AMK), cefoxitin (CFX), and CLA as previously described [19]. As shown in Table 2, all the AMK, CFX, and CLA resistant mutants were sensitive to ETB. ETB showed very close IC values when compared to wild-type Mab subsp. abscessus CIP104536 ${ }^{\mathrm{T}}$, to all AMK, CFX, and CLA resistant mutants. These results demonstrate that ETB is an active compound against wilt-type Mab, as well as against AMK, CFX, and CLA resistant strains. Thus, ETB may be used for treatment in AMK, CFX, and CLA sensitive and resistant Mab infected patients in clinics. To understand ETB in vitro activity better, we also tested ETB in vitro susceptibility test against clinical isolates. The clinical isolate set was organized with $\mathrm{S}$ and $\mathrm{R}$ variants in Mab subsp. abscessus and Mab subsp. massiliense. As shown in Table 2, the growth of Mab clinical isolates was significantly decreased in ETB dose- 
dependent manner. For example, the growth of Mab subsp. abscessus KMRC 00136-61039 S variants and Mab subsp. massiliense KMRC 00200-61202 R variants was inhibited at $\mathrm{IC}_{50}$ values of $0.29 \mu \mathrm{M}$ and $2.75 \mu \mathrm{M}$ of ETB respectively. Most clinical isolates showed growth inhibition at the much lower $\mathrm{IC}_{50}$ range of $0.29-2.75 \mu \mathrm{M}$. Taken altogether, these results indicate that ETB can be considered as an effective drug candidate to cure drug-sensitive and resistant Mab infections regardless of variants in clinic.

Table 2. Inhibitory potency of ETB against Mab subspecies, clinical isolates, and drug resistant Mab strains.

\begin{tabular}{|c|c|c|c|}
\hline \multirow{2}{*}{ Mab Subsp. } & \multirow{2}{*}{ Colony Morphology } & \multicolumn{2}{|c|}{$\begin{array}{l}\text { Cation-Adjusted Mueller-Hinton } \\
\text { (CAMH) Medium }\end{array}$} \\
\hline & & $\mathrm{IC}_{50}(\mu \mathrm{M})$ & $\mathrm{IC}_{90}(\mu \mathrm{M})$ \\
\hline abscessus CIP104536 & $\mathrm{R}$ & 0.25 & 0.56 \\
\hline bolletii CIP108541 & $\mathrm{s}$ & 0.22 & 0.56 \\
\hline massiliense CIP108297 & $\mathrm{s}$ & 0.11 & 0.33 \\
\hline abscessus KMRC 00136-61038 & $\mathrm{s}$ & 1.24 & 2.47 \\
\hline abscessus KMRC 00136-61039 & $\mathrm{s}$ & 0.29 & 1.21 \\
\hline abscessus KMRC 00136-61040 & $\mathrm{R}$ & 2.57 & 10.36 \\
\hline abscessus KMRC 00136-61041 & $\mathrm{S}$ & 0.74 & 1.57 \\
\hline abscessus KMRC 00200-61199 & $\mathrm{s}$ & 1.47 & 4.31 \\
\hline abscessus KMRC 00200-61200 & $\mathrm{S}$ & 0.64 & 2.78 \\
\hline abscessus KMRC 00200-61201 & $\mathrm{S}$ & 0.60 & 1.34 \\
\hline massiliense KMRC 00200-61202 & $\mathrm{R}$ & 2.75 & 4.94 \\
\hline massiliense KMRC 00200-61204 & $\mathrm{s}$ & 1.41 & 2.74 \\
\hline M. abscessus (CLA-R) & $\mathrm{S}$ & 0.31 & 1.17 \\
\hline M. abscessus (AMK-R) & $\mathrm{s}$ & 0.35 & 1.16 \\
\hline M. abscessus (CFX-R) & $\mathrm{S}$ & 0.35 & 0.93 \\
\hline
\end{tabular}

\subsection{Epetraborole Is Non-Toxic to Macrophage and Inhibits Intracellular Growth of Mab}

In order to evaluate the activity of ETB against Mab that reside in macrophages, we tested its potency using a macrophage-based phenotypic assay with an automated cell imaging system. To confirm the colonization by Mab inside a macrophage under treatment with different concentrations of ETB, we used a Mab strain carrying a mWasabi gene which encoded monomeric green fluorescent protein. Using this system, we monitored the intracellular growth of mWasabi protein-expressing Mab subsp. abscessus CIP104536 ${ }^{\mathrm{T}}$ $\mathrm{S}$ variant (hereafter referred to as MabS-mWasabi) in bone marrow-derived macrophages $(\mathrm{mBMDM})$. mBMDM cells were seeded at $7 \times 10^{5} /$ well and infected with MabS-mWasabi that were mixed at a multiplicity of infection (MOI) of 1:1. As shown in Figure 3A, the strong intracellular mWasabi pixel intensity was quantified in DMSO-treated cells (Figure 3A). However, ETB showed significant activity against Mab S-mWasabi replication in $\mathrm{mBMDM}$ in a concentration-dependent manner. The positive control tigecycline (TGC) has also demonstrated good intracellular activity against MabS-mWasabi in a dosedependent manner. The percentage of pixel intensity of intracellular Mab-mWasabi and number of live cells at different concentrations of ETB were quantified. The mBMDM that harbors MabS-mWasabi treated with various concentrations (20, 10, 5, 2.5, 1.3, 0.6, 0.3, and $0.2 \mu \mathrm{M}$ ) of ETB showed significantly reduced mWasabi pixel intensity (Figure 3B). This result demonstrates that ETB has an ability to inhibit bacterial growth inside the cell. 
(A)

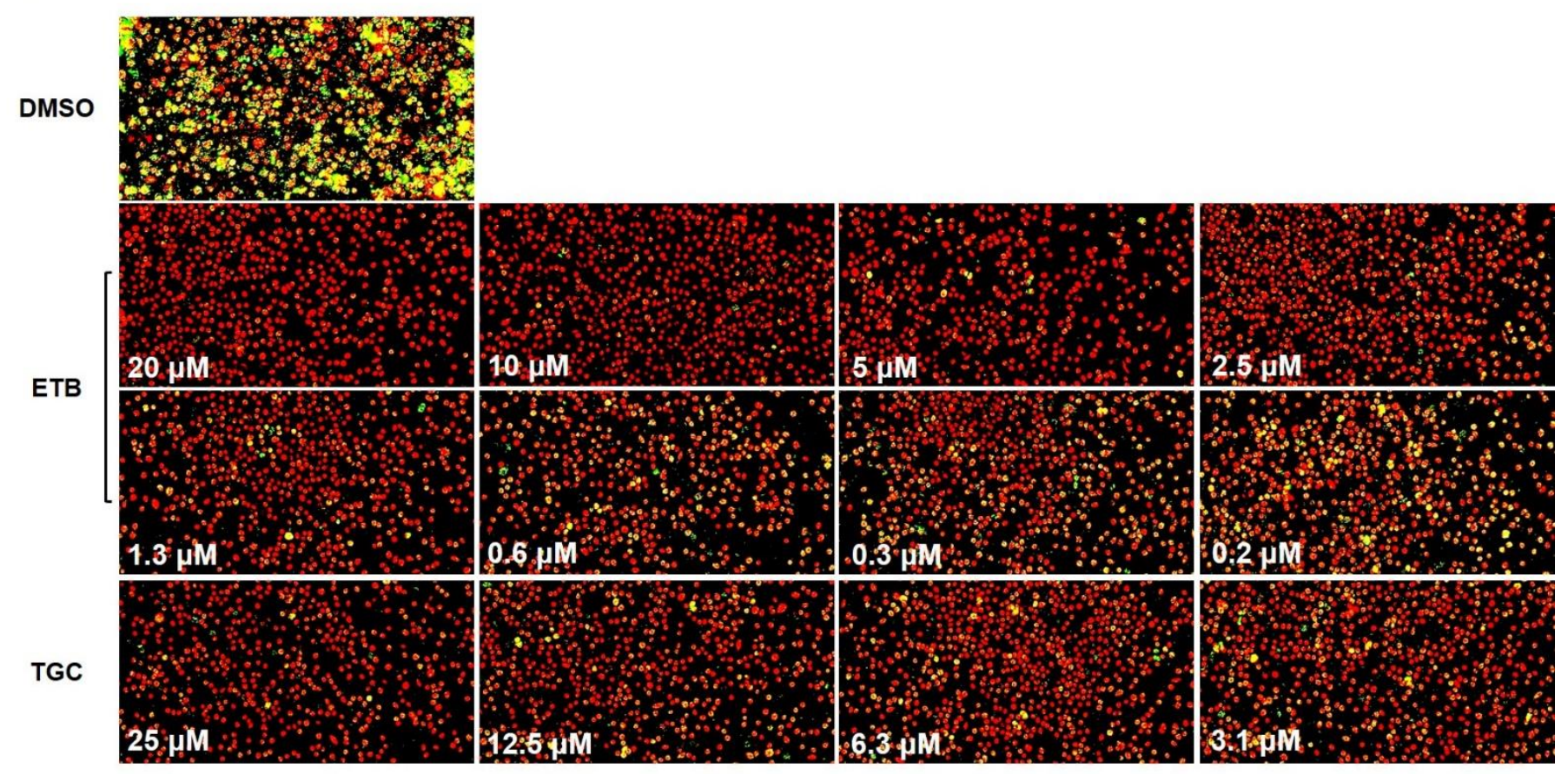

(B)

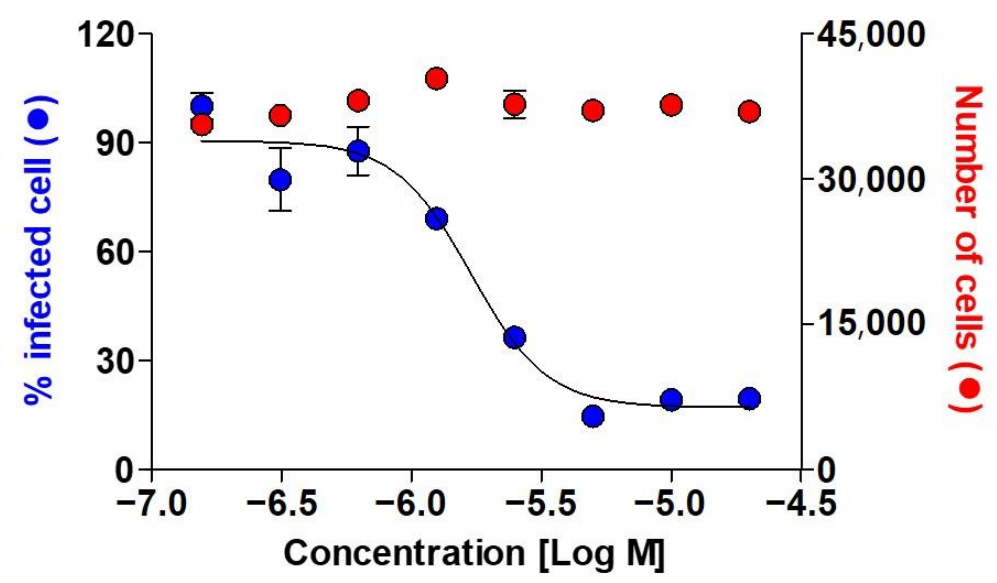

Figure 3. Intracellular activity of ETB against MabS-mWasabi. (A) Images of MabS-mWasabi infected mBMDMs on day 3 after treatment with different doses of ETB and TGC. DMSO was used as negative control. After 3 days of incubation with antibiotics, mBMDMs were stained with syto60 (red), and the cells were analyzed using the automated cell imaging system. The yellow colors represent MabS-mWasabi that were phagocytized by red-stained mBMDM cells. The pixel intensities of live MabS-mWasabi (blue closed circle) and cell number (red closed circle) were quantified after treatment with ETB (B) using CellReporterXpress ${ }^{\circledR}$ Image Acquisition and Analysis Software.

\subsection{ETB Is also Excellent Antimicrobial Agent for Mab in Danio Rerio}

In vivo efficacy of ETB was also validated in Danio rerio (zebrafish; hereafter referred to as ZF) of infection. To determine whether ETB has the ability to treat Mab infected $\mathrm{ZF}$ as a therapeutic agent. ETB in vivo efficacy was evaluated in ZF after infection with Mab subsp. abscessus CIP104536 ${ }^{\mathrm{T}} \mathrm{R}$ variant that express mWasabi green fluorescence (hereafter referred to as MabR-mWasabi) at concentrations of 6.25, 12.5, 25, and $50 \mu \mathrm{M}$. First, we investigated whether the mWasabi fluorescent signal in infected ZF would be reduced by adding ETB in a dose-dependent manner. For this, dissemination of MabRmWasabi in ZF was monitored under a fluorescence microscope according to ETB con- 
centrations. After the MabR-mWasabi infection, ETB was administered at each different concentration $(6.25,12.5,25$, and $50 \mu \mathrm{M})$ for up to 5 days post-infection (dpi). As shown in Figure 4A, MabR-mWasabi was disseminated and localized inside of ZF, especially in the head when the DMSO was treated. However, the mWasabi fluorescent signal was significantly reduced in the ETB treated condition. In more detail, a significant mWasabi reduction in the MabR-mWasabi infected ZF head was observed at $25 \mu \mathrm{M}$ ETB. Furthermore, almost no mWasabi protein signals were detected in the ZF when MabR-mWasabi infected ZF were treated with 25 and $50 \mu \mathrm{M}$ ETB. This mWasabi protein signal reduction at ETB $50 \mu \mathrm{M}$ was similar to those of the positive control TGC treatment at $50 \mu \mathrm{M}$. To determine whether MabR proliferated in the ZF body after treatment of ETB in different doses, each infected ZF was crushed, sampled and the bacterial burden was enumerated on a 7H10 Middlebrook agar plate. Figure 4A shows that MabR could colonize the ZF and replicate inside the host and that the CFU showed significant differences between DMSO control and ETB treated groups. Until $6.25 \mu \mathrm{M}$ of ETB treatment, there was no significant bacterial colony reduction on the 7H10 agar plate. However, after $12.5 \mu \mathrm{M}$ of ETB treatment there started to be a reduction in the bacterial number. The smallest bacterial populations per ZF were found to occur in the presence of ETB $50 \mu \mathrm{M}$. This led to a $3.8 \log _{10}$ reduction when compared to DMSO control.

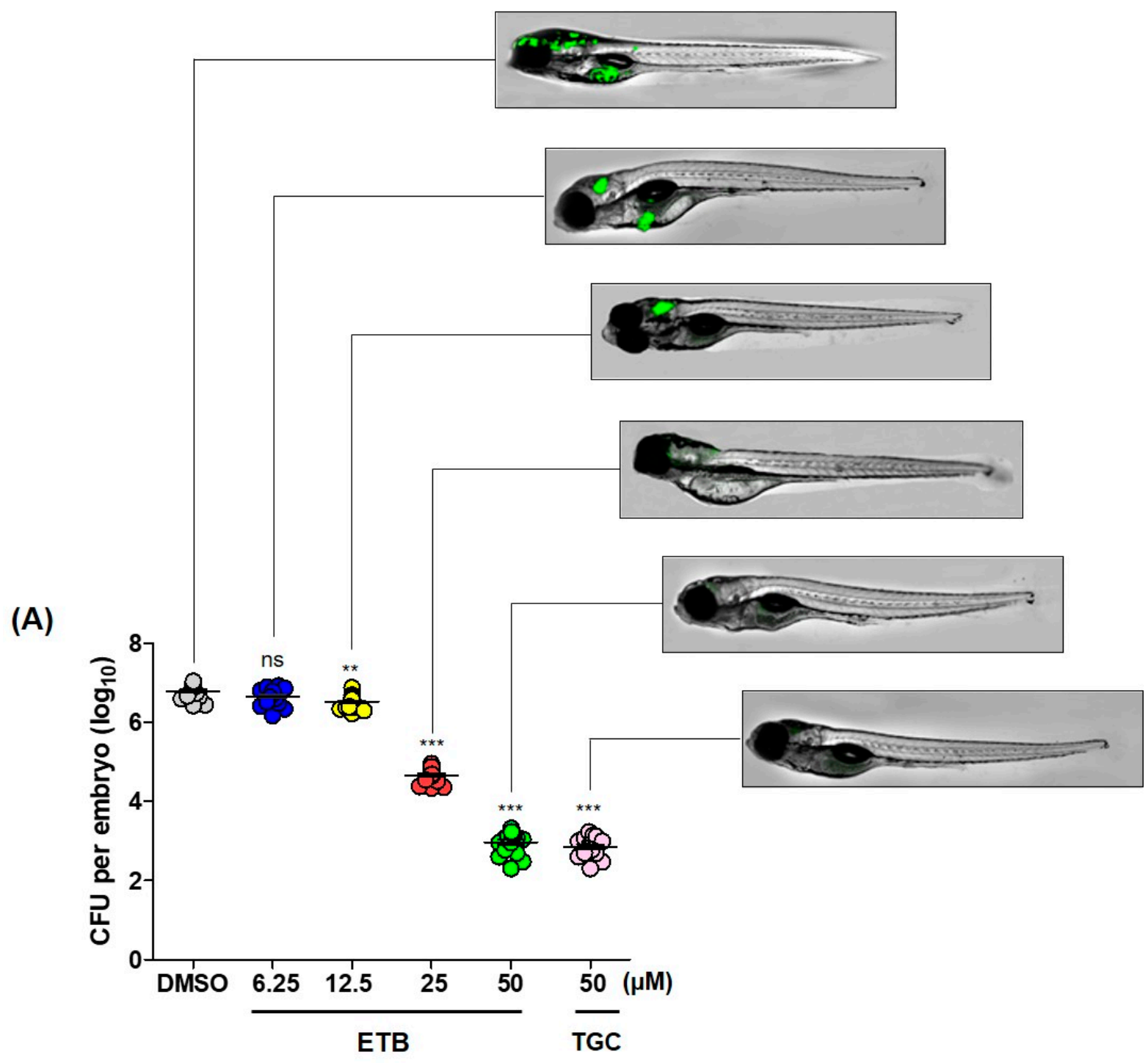

Figure 4. Cont. 
(B)

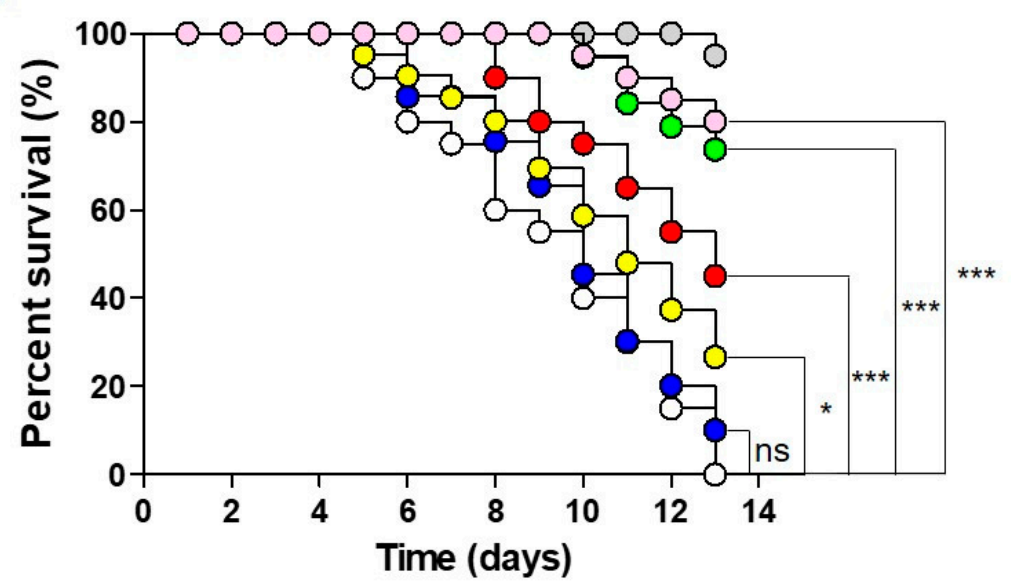

-O- DMSO

-O- Inf UNT

$\rightarrow$ ETB 6.25

-O- ETB 12.5

-O- ETB 25

-O- ETB 50

-O- TGC 50

Figure 4. ZF In vivo efficacy of ETB. (A) Different concentrations of ETB $(6.25,12.5,25$, and $50 \mu \mathrm{M})$ and TGC (50 $\mu \mathrm{M})$ were treated to the ZF infected with MabR-mWasabi and proliferation or reduction of mWasabi signal in ZF was monitored under the fluorescent microscope. After antibiotics treatment, the bacterial burden of infected ZF was quantified through traditional agar plate quantification method. Data was expressed as the mean log 10 CFU per embryo $(n=10$ of each condition) from three independent experiments. (B) All infected fishes treated at 6.25, 12.5, 25, and $50 \mu \mathrm{M}$ of ETB and TGC $(50 \mu \mathrm{M})$. Survival curve was plotted from MabR-mWasabi infected ZF for 13 days. $(n=20$, representative of three independent experiments). Survival curves were compared with the log-rank (Mantel-Cox) test ${ }^{*} p<0.05,{ }^{* *} p<0.01$; *** $p<0.001$; ns: Not significant). Inf UNT: Infected but not treated control.

Next, we evaluated the survival rate of MabR infected ZF to know whether ETB could extend the lifespan of infected ZF after treatment of ETB dose-dependently. The survival rates of MabR infected ZFs were observed for 13 days while the tank water was replaced using $6.25,12.5,25$, and $50 \mu \mathrm{M}$ of ETB daily. $50 \mu \mathrm{M}$ of TGC treatment was used as a positive control and untreated ZF group was used as a negative control. ZF without MabR infection was used as a mock control. The results shown in Figure $4 \mathrm{~B}$ present the survival rates of the ETB-treated ZF cultures infected with MabR compared with those of the untreated control ZF. $100 \%$ of fish that were infected by MabR in the untreated group died at 13 days post infection (dpi). However, the ETB treated ZF group showed significantly extended lifespans. When MabR infected ZF was exposed to 6.25 and $12.5 \mu \mathrm{M}$ of ETB for 13 days, around 75 to $90 \%$ of the infected $\mathrm{ZF}$ died, similarly with the untreated group. However, when we increased the dosage of ETB to 25 and $50 \mu \mathrm{M}$, exponential extended lifespan was observed. The TGC (50 $\mu \mathrm{M})$ treated ZF group also showed a similar significant lifespanextending effect as was observed in the $50 \mu \mathrm{M}$ ETB treatment group. Taken together, these results suggest that ETB has a therapeutic effect against Mab in vivo.

\section{Discussion}

In this study, we screened the Pandemic Response Box that consists of a set of 400 structurally diverse compounds. Using this chemical library, we have conducted dual screening with both Mab subsp. abscessus CIP $104536^{\mathrm{T}} \mathrm{S}$ - and R-variants. S-variants possess glycopeptidolipids (GPL) in the mycobacterial cell wall and minimal trehalose dimycolate (TDM) which contributes to the Mycobacterium tuberculosis virulence [18]. However, GPL is noticeably absent in R-variants and these R-variants are more virulent than S-variants in the animal model of infection by causing invasive infection. [20,21]. The R-variants could emerge from CF patients chronically colonized with an S strain, creating a more aggressive, invasive pulmonary infection. Thus, identification of a new compound that has more potency to the R-variant would be a beneficial to the patients who are suffering from R-variant infection. From the screen, we narrowed down 3 different hits named ETB, ERV, and BDQ that showed potent activity to both S and R-variants. 
ERV is a synthetic halogenated tetracycline class and has a broad spectrum of activity against aerobic and anaerobic Gram-negative and Gram-positive bacteria [22]. ERV disrupts bacterial protein synthesis via binding to the $30 \mathrm{~S}$ ribosomal subunit, thus preventing the incorporation of amino acid residues into elongating peptide chains [23]. In recent susceptibility tests against Mab, ERV showed 2-4 times lower in vitro MIC (0.125-2 mg/L) than tigecycline. Furthermore, ERV has improved intravenously administered pharmacokinetic/pharmacodynamic parameters and it is suggested that ERV could be more efficacious clinically than tigecycline [24].

BDQ was approved by the Food and Drug Administration and the European Medicines Agency for the treatment of multidrug-resistant tuberculosis (MDR-TB). It is a diarylquinoline antibiotic that targets the essential $F_{0} F_{1}$ ATP synthase [25,26]. BDQ has shown favorable in vivo activity against Mab infected animal models such as in ZF and the immunocompromised $[27,28]$. However, the efficacy of BDQ is somewhat doubtful and may depend on the animal model. For instance, BDQ did not decrease the bacterial burden after one month of treatment in a nude mouse that was athymic with a depletion of $T$ cells [29]. In addition, Philley et al. reported preliminary effectiveness of BDQ-containing regimen treatment as salvage therapy for patients who have Mab lung disease. However, after 6 months of observation, only one patient among six showed an improvement of clinical symptoms [30]. Therefore, there is no clinical evidence that BDQ is a potential agent in the treatment of Mab infections.

In this study, we evaluated the activity of ETB in different infection models and it showed an excellent therapeutic effect against Mab. As shown in Tables 1 and 2, survival of $M a b$ was greatly reduced after ETB treatment in all the Mab strains tested in vitro, including Mab CIP $104536^{\mathrm{T}} \mathrm{S}$ - and R-variants and clinical isolates at a $\mathrm{IC}_{50}$ range of $0.29-2.75 \mu \mathrm{M}$. Furthermore, ETB could successfully inhibit spontaneously-induced drug-resistant $\mathrm{Mab}$ CIP $104536^{\mathrm{T}} \mathrm{S}$ strains that were generated previously [19]. In a time-kill assay, ETB exhibited dramatic concentration-dependent killing against Mab CIP $104536^{\mathrm{T}} \mathrm{S}$ at all tested drug concentrations $(0.5-5 \times$ IC). Bacterial regrowth was not observed until 5 days after ETB treatment.

This in vitro activity was further validated using the mBMDMs model of infection. The intracellular activity of ETB against Mab, which resides in mBMDMs, was assessed using the Automated Cell Imaging System. The mBMDMs cells were infected with MabSmWasabi and the number of fluorescent $\mathrm{mWasabi}$ signals was statistically enumerated after treatment of ETB in a concentration-dependent manner. Using this system, we evaluated the intracellular activity and cytotoxicity of ETB in mBMDMs at the same time. As shown in Figure 3A, ETB showed significant fluorescent $M a b$ reduction in a dose-dependent manner. ETB could completely inhibit fluorescence expressing Mab signals at $2.5 \mu \mathrm{M}$ without cytotoxicity. $\mathrm{IC}_{50}$ of ETB against intracellular Mab was $1.7 \mu \mathrm{M}$ (Figure 3B). This result showed comparable activity to TGC, which has bactericidal intracellular effect on Mab infecting THP-1 macrophages (Figure 4B) [31]. Thus, we demonstrated that ETB can successfully inhibit the growth of intracellular Mab without cytotoxicity.

The potent in vitro and intracellular inhibitory activity of ETB was further tested in the ZF infection model. ZF embryo caudal vein injection model is a popular tractable in vivo model that is often used to generate systemic infections for subsequent analyses, including survival experiments and bacterial burden determination [32]. This model has been successively used for in vivo anti-bacterial drug evaluations for Mab and Mycobacterium marinum [9,33-37]. For this reason, the ZF embryo infection model has been used as a preliminary in vivo efficacy evaluation before proceeding to a mouse in vivo efficacy evaluation in the drug discovery pipeline. Thus, we also evaluated the in vivo efficacy of ETB by injecting MabR variant into ZF through caudal vein injection. In order to evaluate the toxicity of ETB, we evaluated the maximum tolerated dose (MTD) of ETB to ZF. Fifteen ZF were used in each treatment group. A broad range of ETB (ranging from 6.25 to $100 \mu \mathrm{M}$ ) was added to ZF-containing fish water, without bacterial infection. Around $100 \%$ of ZF died after 12 days of exposure to $100 \mu \mathrm{M}$ ETB. Thus, less than $50 \mu \mathrm{M}$ of ETB, which 
does not show significant lethality, were used for in vivo efficacy tests (data not shown). In vivo efficacy test, ETB treatment showed significant bacterial CFU reduction inside infected ZF in a dose-dependent manner in comparison with TGC treatment (Figure 4A). Furthermore, MabR resulted in $100 \%$ of ZF death infected at 13 days post infection (dpi), while $50 \mu \mathrm{M}$ ETB treatment resulted in a significantly enhanced lifespan. However, it is important to mention that ZF embryos model of infection also has some limitations. Firstly, adaptive immunity in ZF embryo is not fully matured until after 4-6 weeks [38,39]. Thus, it is likely to generate different outcomes than those obtained in mammalian models. Therefore, to show the effectiveness of the compound in a chronic model, it would be better to use immunocompromised mice which can allow sustained Mab pulmonary infection [40]. Second, some compounds have a capacity to persistently adhere to the ZF skin during the treatment procedure resulting in an artificial carryover effect in the evaluation of in vivo efficacy through bacterial CFU quantification [36]. Moreover, pharmacokinetics are not known in ZF which makes it difficult to directly transpose the treatment dose obtained from ZF to humans [35]. Thus, a drug efficacy evaluating system using ZF-Mab infection should be regarded as an early model for screening new bio-active compounds using the chemical library or preclinical drug testing. These should then be evaluated in other higher animal models before clinical trials.

ETB is a benzoxaboroles analogue and leucyl-tRNA synthetase (LeuRS) inhibitor and it has a novel mode of action against Gram-negative bacterial infection such as urinary tract infections [41,42]. The LeuRS catalyzes the ATP-dependent ligation of L-leucine to tRNA. In previous studies, the benzoxaborole analogue (referred as compound 1) also showed antitubercular activity against $M$. tuberculosis (MIC of $0.26 \mu \mathrm{g} / \mathrm{mL}$ ) and M. smegmatis (MIC of $1 \mu \mathrm{g} / \mathrm{mL}$ ) [43]. Furthermore, a spontaneous mutant was generated from both species on a Middlebrook $7 \mathrm{H} 10$ agar plate containing benzoxaborole analogue at $5 \times$ or $10 \times \mathrm{MIC}_{99}$ and spontaneous mutants were shown to be harboring an amino acid substitution on mycobacterial LeuRS. Intriguingly, Mab also contains leuS (MAB_4923c) which has a 75\% and $78 \%$ amino acid homology with $M$. tuberculosis and M. smegmatis respectively. Thus, ETB might share the same mechanism of action with the benzoxaborole analogue (compound 1) which has demonstrated its activity against $M$. tuberculosis and M. smegmatis. Based on ClinicalTrials.gov in 2017, a clinical phase II study of ETB for the treatment of complicated urinary tract infection and complicated intra-abdominal infection was terminated due to the rapid emergence of drug resistance during treatment [41]. Recently however, a new oxaborole inhibitor, DS86760016, has reignited the re-use of the LeuRS inhibitor resulting in a lower frequency of resistance development than ETB in comparative murine urinary tract infection models. Unfortunately, DS86760016 is not commercially available currently because Daiichi Sankyo India, the pharmaceutical company that discovered DS86760016, was closed in 2017 (personal communication with Dr. Nobuhisa Masuda). For this reason, we synthesized DS86760016 through a collaboration with the medicinal chemistry team based on the structure-activity relationship. Currently, we have checked its activity for $M a b$ and it showed similar IC value with ETB against Mab (data not shown). The newly synthesized DS86760016 will be further tested in several models of infection to know its anti-Mab activity and resistant mutant frequency in comparison with ETB soon.

In this study, we screened the Pandemic Response Box and identified several hits in the concentration-response curve. Among these hits, ETB showed excellent in vitro activity against 3 different $M a b$ subspecies, various clinical isolates, and drug resistant strains. In addition, ETB showed significant intracellular Mab killing activity at low concentrations. It also showed a terrific therapeutic effect in the Mab infected ZF models. Therefore, ETB is a potential anti-Mab drug candidate that can be further developed for the anti-Mab drug discovery pipeline. 


\section{Materials and Methods}

\subsection{Bacterial Strains and Culture Conditions}

Mab subsp. abscessus CIP $104536^{\mathrm{T}}$ S- and R-variants were kindly provided by Dr. Laurent Kremer (CNRS, IRIM, Universite' de Montpellier, Montpellier, France). Mab subsp. bolletii CIP108541 ${ }^{\mathrm{T}}$ and Mab subsp. massiliense CIP108297 ${ }^{\mathrm{T}}$ were obtained from the Collection de l'Institut Pasteur (CIP, Paris, France). Clinical isolates were purchased from the Korea Mycobacterium Resource Center (KMRC, Osong, Korea). Mab strains were grown in Middlebrook 7H9 broth (BD Biosciences, San Hose, CA, USA) supplemented with 10\% (v:v) albumin, dextrose, and catalase (ADC; BD), $0.2 \%$ (v:v) glycerol and $0.05 \%$ $(v: v)$ Tween 80 or Middlebrook 7H10 agar plates (BD) containing 10\% (v:v) oleic acid and ADC (OADC; BD) enrichment and 0.5\% (v:v) glycerol. All cultures were grown at $37^{\circ} \mathrm{C}$ with shaking at $180 \mathrm{rpm}$. CLA and ETB were purchased from Sigma-Aldrich (St. Louis, MO, USA) and MedKoo Biosciences, Inc. (Cat\#: 319569; Morrisville, NC, USA) respectively. TGC was purchased from Adooq Bioscience (Cat\#: A10933; Irvine, CA, USA). For the mBMDM preparation and ZF infection, recombinant Mab CIP $104536^{\mathrm{T}} \mathrm{S}$ and $\mathrm{R}$ that carrying a pMV262-mWasabi plasmid expressing mWasabi protein was prepared as previously described [33].

\subsection{Resazurin Microtiter Assay (REMA)}

Fifty microliters of Mab culture (approximately $5 \times 10^{4} \mathrm{CFU} / \mathrm{mL}$ ) in Middlebrook 7H9 broth supplemented with $10 \%$ ADC, $0.2 \%$ glycerol was used per well and $50 \mu \mathrm{L}$ of serial 2-fold dilutions of test compound solution was added to each well of a sterile, polystyrene 96-well cell culture plate (SPL; Gyeonggi-do, Korea). A drug-free growth control was included in each plate. $200 \mu \mathrm{L}$ of sterile water was added to outer perimeter wells to prevent evaporation during incubation. Plates were then covered with self-adhesive membranes and incubated at $37{ }^{\circ} \mathrm{C}$ for 3 days. The resazurin solution was prepared as a $0.025 \%(w / v)$ solution in sterile distilled water using resazurin sodium salt powder (Sigma, St. Louis, MO, USA), filter sterilized. After 3 days of incubation, $40 \mu \mathrm{L}$ of the resazurin solution was added to the wells. Fluorescence was measured using a SpectraMax ${ }^{\circledR}$ M3 Multi-Mode Microplate Reader (Molecular Devices, Sunnyvale, CA, USA). Dose response curve was constructed and concentrations required to inhibit bacterial growth by $50 \%$ (IC50) was determined the GraphPad Prism software (version 6.05; San Diego, CA, USA).

\subsection{ETB Time-Kill Assay for Mab}

An early exponential phase mycobacterial culture $\left(10^{7} \mathrm{CFU} / \mathrm{mL}\right)$ was prepared in $30 \mathrm{~mL}$ of $7 \mathrm{H} 9$ broth supplemented with $10 \%$ ADC, $0.2 \%$ glycerol. Two-fold increasing concentrations of ETB (from 0.5 to $5 \times$ MIC), $5 \times$ MIC of CLA and a drug-free growth control was used. To perform CFU counting, $100 \mu \mathrm{L}$ samples were taken from each bottle, and 10-fold dilutions of samples were then made in PBS (900 $\mu \mathrm{L}$ PBS, $100 \mu \mathrm{L}$ sample) at different time intervals $(0,1,2,3,4$, and 5 days). A total of $50 \mu \mathrm{L}$ of each dilution was plated onto $7 \mathrm{H} 10$ agar, containing 10\% OADC supplement and bactericidal activity of ETB was compared to the drug-free growth control. All time-kill experiments were performed in triplicate and the mean CFU counts plotted.

\subsection{Intracellular Bacterial Replication Assays}

Intracellular activity of ETB was analyzed as previously described [44]. Briefly, differentiated mBMDMs $\left(7 \times 10^{5}\right.$ cells/well $)$ cells were infected with MabS-mWasabi at a multiplicity of infection (MOI) of 1:1. After $3 \mathrm{~h}$ of infection, the cells were washed with PBS (phosphate-buffered saline) and incubated with DMEM (Dulbecco's Modified Eagle's medium) containing $250 \mu \mathrm{g} / \mathrm{mL}$ amikacin for $1 \mathrm{~h}$ to kill the extracellular MabSmWasabi [45]. The cells were washed with PBS and then dispensed into 96-well plates (Corning, New York, NY, USA). The broth medium, with a 2-fold serial dilution of compound, was subjected to a 96-well plate. For all experiments, the amount of DMSO was maintained at a $1 \%$ final concentration per well. After 3 days of incubation at $37^{\circ} \mathrm{C}$ and 
$5 \% \mathrm{CO}_{2}$, macrophages were stained for $1 \mathrm{~h}$ with $5 \mu \mathrm{M}$ Syto 60 dye (Thermo Fisher Scientific, Waltham, MA, USA), and images were recorded using the ImageXpress Pico Automated Cell Imaging System (Molecular Devices, Sunnyvale, CA, USA). The number of bacteria in macrophage and macrophage number were quantified using the CellReporterXpress ${ }^{\circledR}$ Image Acquisition and Analysis Software (Molecular Devices).

\subsection{Drug Efficacy Assessment in MabR-mWasabi Infected ZF}

ZF experiments were approved by the Animal Research Ethics Committee of Gyeongsang National University (Project identification code: GNU-190325-E0014, Approval date: 25 March 2019). ZF larvae at 30-48 h post-fertilization were dechorionated and tricaine $(270 \mathrm{mg} / \mathrm{L})$ was used for ZF larvae anesthesia. Mid-log-phase cultures of MabR-mWasabi were used for ZF larvae infection as described previously [35]. Around $3 \mathrm{~nL}$ of MabR-mWasabi (400 CFU) was injected via caudal vein using a Tritech Research Digital microINJECTOR ${ }^{\mathrm{TM}}$, (MINJ-D; Tritech research, CA, USA). The accurate infected CFU was enumerated by CFU quantification method using 7H10 agar plates containing $10 \%$ OADC enrichment and $0.5 \%$ glycerol with kanamycin $(50 \mathrm{mg} / \mathrm{L})$. The infected larvae were transferred into 96-well plates (2 fishes/well) and incubated at $28.5^{\circ} \mathrm{C}$ after treatment of compound. Different concentration of ETB (ranges from 6.25 to $50 \mu \mathrm{M}$ at the final concentration) and TGC $(50 \mu \mathrm{M})$ were added directly into the blue fish water (methylene blue $300 \mu \mathrm{L} / \mathrm{L}$ ). The fish water and compound were renewed once daily and each different concentration of compound was absorbed orally by infected larvae. The embryos survival was monitored and dead embryos (no heartbeat) were recorded on daily to determine survival curve. The DMSO was used as negative. The in vivo efficacy of ETB was evaluated by quantification of $M a b R$ load in ZF by counting serial dilutions of bacterial CFU as previous described $[9,33,44]$ The CFU quantification and survival curve were plotted by GraphPad Prism software using the method from Kaplan and Meier, and log-rank (Mantel-Cox) test respectively.

Author Contributions: J.J., J.-W.P. and K.S. designed the study and experiments. T.K., B.-T.-B.H., N.Q., B.H., Y.P., S.J. and J.S. performed the experiments and generated and documented the data. J.J. analyzed the data and wrote the manuscript. All authors have read and agreed to the published version of the manuscript.

Funding: This research was supported by the National Research Foundation of Korea (grants 2020R1A2C1004077).

Institutional Review Board Statement: The study was conducted according to the guidelines of the Animal Research Ethics Committee of Gyeongsang National University (Project identification code: GNU-190325-E0014, Approval date: 25 Mar 2019).

Informed Consent Statement: Not applicable.

Acknowledgments: T.K., B.-T.-B.H., N.Q., B.H., Y.P. and J.S. were supported by a scholarship from the BK21 Four Program, the Ministry of Education and Human Resources, Korea. MMV and DNDi for providing the Pandemic Response Box.

Conflicts of Interest: The authors declare no conflict of interest.

\section{References}

1. Bentur, L.; Gur, M.; Ashkenazi, M.; Livnat-Levanon, G.; Mizrahi, M.; Tal, A.; Ghaffari, A.; Geffen, Y.; Aviram, M.; Efrati, O. Pilot study to test inhaled nitric oxide in cystic fibrosis patients with refractory Mycobacterium abscessus lung infection. J. Cyst. Fibros. 2019, 19, 225-231. [CrossRef]

2. Hunt-Serracin, A.C.; Parks, B.J.; Boll, J.; Boutte, C.C. Mycobacterium abscessus cells have altered antibiotic tolerance and surface glycolipids in artificial cystic fibrosis sputum medium. Antimicrob. Agents Chemother. 2019, 63, e02488-18. [CrossRef]

3. Ferro, B.E.; Srivastava, S.; Deshpande, D.; Pasipanodya, J.G.; Van Soolingen, D.; Mouton, J.W.; Van Ingen, J.; Gumbo, T. Failure of the amikacin, cefoxitin, and clarithromycin combination regimen for treating pulmonary Mycobacterium abscessus infection. Antimicrob. Agents Chemother. 2016, 60, 6374-6376. [CrossRef]

4. Jarand, J.; Levin, A.; Zhang, L.; Huitt, G.; Mitchell, J.D.; Daley, C.L. Clinical and microbiologic outcomes in patients receiving treatment for Mycobacterium abscessus pulmonary disease. Clin. Infect. Dis. 2011, 52, 565-571. [CrossRef] [PubMed] 
5. Chopra, S.; Matsuyama, K.; Hutson, C.; Madrid, P. Identification of antimicrobial activity among FDA-approved drugs for combating Mycobacterium abscessus and Mycobacterium chelonae. J. Antimicrob. Chemother. 2011, 66, 1533-1536. [CrossRef] [PubMed]

6. Malin, J.J.; Winter, S.; Van Gumpel, E.; Plum, G.; Rybniker, J. Extremely low hit rate in a diverse chemical drug screen targeting mycobacterium abscessus. Antimicrob. Agents Chemother. 2019, 63, e01008-19. [CrossRef] [PubMed]

7. Gupta, R.; Netherton, M.; Byrd, T.F.; Rohde, K.H. Reporter-based assays for high-throughput drug screening against Mycobacterium abscessus. Front. Microbiol. 2017, 8, 2204. [CrossRef] [PubMed]

8. Jeong, J.; Kim, G.; Moon, C.; Kim, H.J.; Kim, T.H.; Jang, J. Pathogen Box screening for hit identification against Mycobacterium abscessus. PLoS ONE 2018, 13, e0195595. [CrossRef] [PubMed]

9. Hanh, B.T.B.; Park, J.W.; Kim, T.H.; Kim, J.S.; Yang, C.S.; Jang, K.; Cui, J.; Oh, D.C.; Jang, J. Rifamycin O, An Alternative Anti-Mycobacterium abscessus Agent. Molecules 2020, 25, 1597. [CrossRef] [PubMed]

10. Moreira, W.; Lim, J.J.; Yeo, S.Y.; Ramanujulu, P.M.; Dymock, B.W.; Dick, T. Fragment-based whole cell screen delivers hits against M. tuberculosis and non-tuberculous mycobacteria. Front. Microbiol. 2016, 7, 1392. [CrossRef] [PubMed]

11. Low, J.L.; Wu, M.L.; Aziz, D.B.; Laleu, B.; Dick, T. Screening of TB actives for activity against nontuberculous mycobacteria delivers high hit rates. Front. Microbiol. 2017, 8, 1539. [CrossRef]

12. Berube, B.J.; Castro, L.; Russell, D.; Ovechkina, Y.; Parish, T. Novel screen to assess bactericidal activity of compounds against non-replicating mycobacterium abscessus. Front. Microbiol. 2018, 9, 2417. [CrossRef] [PubMed]

13. Gutiérrez, A.V.; Viljoen, A.; Ghigo, E.; Herrmann, J.L.; Kremer, L. Glycopeptidolipids, a double-edged sword of the Mycobacterium abscessus complex. Front. Microbiol. 2018, 9, 1145. [CrossRef]

14. Viljoen, A.; Viela, F.; Kremer, L.; Dufrêne, Y.F. Fast chemical force microscopy demonstrates that glycopeptidolipids define nanodomains of varying hydrophobicity on mycobacteria. Nanoscale Horiz. 2020, 5, 944-953. [CrossRef]

15. Richter, A.; Shapira, T.; Av-Gay, Y. THP-1 and Dictyostelium Infection Models for Screening and Characterization of AntiMycobacterium abscessus Hit Compounds. Antimicrob. Agents Chemother. 2020, 64, e01601-19. [CrossRef]

16. Luthra, S.; Rominski, A.; Sander, P. The role of antibiotic-target-modifying and antibiotic-modifying enzymes in mycobacterium abscessusdrug resistance. Front. Microbiol. 2018, 9, 2179. [CrossRef]

17. Loots, D.T. New insights into the survival mechanisms of rifampicin-resistant Mycobacterium tuberculosis. J. Antimicrob. Chemother. 2016, 71, 392-407. [CrossRef] [PubMed]

18. Johansen, M.D.; Herrmann, J.L.; Kremer, L. Non-tuberculous mycobacteria and the rise of Mycobacterium abscessus. Nat. Rev. Microbiol. 2020, 18, 392-407. [CrossRef] [PubMed]

19. Kim, T.S.; Choe, J.H.; Kim, Y.J.; Yang, C.S.; Kwon, H.J.; Jeong, J.; Kim, G.; Park, D.E.; Jo, E.K.; Cho, Y.L.; et al. Activity of LCB01-0371, a novel oxazolidinone, against mycobacterium abscessus. Antimicrob. Agents Chemother. 2017, 61, e02752-16. [CrossRef]

20. Catherinot, E.; Clarissou, J.; Etienne, G.; Ripoll, F.; Emile, J.F.; Daffé, M.; Perronne, C.; Soudais, C.; Gaillard, J.L.; Rottman, M. Hypervirulence of a rough variant of the Mycobacterium abscessus type strain. Infect. Immun. 2007, 75, 1055-1058. [CrossRef]

21. Bernut, A.; Herrmann, J.L.; Kissa, K.; Dubremetz, J.F.; Gaillard, J.L.; Lutfalla, G.; Kremer, L. Mycobacterium abscessus cording prevents phagocytosis and promotes abscess formation. Proc. Natl. Acad. Sci. USA 2014, 111, E943-E952. [CrossRef]

22. Sutcliffe, J.A.; O’Brien, W.; Fyfe, C.; Grossman, T.H. Antibacterial activity of eravacycline (TP-434), a novel fluorocycline, against hospital and community pathogens. Antimicrob. Agents Chemother. 2013, 57, 5548-5558. [CrossRef] [PubMed]

23. Scott, L.J. Eravacycline: A Review in Complicated Intra-Abdominal Infections. Drugs 2019, 79, 315-324. [CrossRef]

24. Kaushik, A.; Ammerman, N.C.; Martins, O.; Parrish, N.M.; Nuermberger, E.L. In vitro activity of new tetracycline analogs omadacycline and eravacycline against drug-resistant clinical isolates of mycobacterium abscessus. Antimicrob. Agents Chemother. 2019, 63, e00470-19. [CrossRef] [PubMed]

25. Cohen, J. Approval of novel TB drug celebrated-With restraint. Science 2013, 339, 130. [CrossRef] [PubMed]

26. Matteelli, A.; Carvalho, A.C.C.; Dooley, K.E.; Kritski, A. TMC207: The first compound of a new class of potent anti-tuberculosis drugs. Future Microbiol. 2010, 5, 849-858. [CrossRef] [PubMed]

27. Dupont, C.; Viljoen, A.; Thomas, S.; Roquet-Banères, F.; Herrmann, J.L.; Pethe, K.; Kremer, L. Bedaquiline inhibits the ATP synthase in mycobacterium abscessus and is effective in infected zebrafish. Antimicrob. Agents Chemother. 2017, 61, e01225-17. [CrossRef]

28. Obregón-Henao, A.; Arnett, K.A.; Henao-Tamayo, M.; Massoudi, L.; Creissen, E.; Andries, K.; Lenaerts, A.J.; Ordway, D.J. Susceptibility of mycobacterium abscessus to antimycobacterial drugs in preclinical models. Antimicrob. Agents Chemother. 2015, 59, 6904-6912. [CrossRef]

29. Lerat, I.; Cambau, E.; Roth Dit Bettoni, R.; Gaillard, J.L.; Jarlier, V.; Truffot, C.; Veziris, N. In vivo evaluation of antibiotic activity against Mycobacterium abscessus. J. Infect. Dis. 2014, 209, 905-912. [CrossRef]

30. Philley, J.V.; Wallace, R.J.; Benwill, J.L.; Taskar, V.; Brown-Elliott, B.A.; Thakkar, F.; Aksamit, T.R.; Griffith, D.E. Preliminary results of bedaquiline as salvage therapy for patients with nontuberculous mycobacterial lung disease. Chest 2015, 148, 499-506. [CrossRef]

31. Molina-Torres, C.A.; Tamez-Peña, L.; Castro-Garza, J.; Ocampo-Candiani, J.; Vera-Cabrera, L. Evaluation of the intracellular activity of drugs against Mycobacterium abscessus using a THP-1 macrophage model. J. Microbiol. Methods 2018. [CrossRef] 
32. Bernut, A.; Dupont, C.; Sahuquet, A.; Herrmann, J.L.; Lutfalla, G.; Kremer, L. Deciphering and imaging pathogenesis and cording of Mycobacterium abscessus in zebrafish embryos. J. Vis. Exp. 2015, 53130. [CrossRef] [PubMed]

33. Kim, T.H.; Bich Hanh, B.T.; Kim, G.; Lee, D.G.; Park, J.W.; Lee, S.E.; Kim, J.S.; Kim, B.S.; Ryoo, S.; Jo, E.K.; et al. Thiostrepton: A Novel Therapeutic Drug Candidate for Mycobacterium abscessus Infection. Molecules 2019, 24, 4511. [CrossRef] [PubMed]

34. Takaki, K.; Davis, J.M.; Winglee, K.; Ramakrishnan, L. Evaluation of the pathogenesis and treatment of Mycobacterium marinum infection in zebrafish. Nat. Protoc. 2013, 8, 1114-1124. [CrossRef] [PubMed]

35. Bernut, A.; Le Moigne, V.; Lesne, T.; Lutfalla, G.; Herrmann, J.L.; Kremer, L. In Vivo assessment of drug efficacy against Mycobacterium abscessus using the embryonic zebrafish test system. Antimicrob. Agents Chemother. 2014, 58, 4054-4063. [CrossRef]

36. Ordas, A.; Raterink, R.J.; Cunningham, F.; Jansen, H.J.; Wiweger, M.I.; Jong-Raadsen, S.; Bos, S.; Bates, R.H.; Barros, D.; Meijer, A.H.; et al. Testing tuberculosis drug efficacy in a zebrafish high-throughput translational medicine screen. Antimicrob. Agents Chemother. 2015, 59, 753-762. [CrossRef]

37. Bernut, A.; Herrmann, J.L.; Ordway, D.; Kremer, L. The diverse cellular and animal models to decipher the physiopathological traits of Mycobacterium abscessus infection. Front. Cell. Infect. Microbiol. 2017, 7, 100. [CrossRef]

38. Lam, S.H.; Chua, H.L.; Gong, Z.; Lam, T.J.; Sin, Y.M. Development and maturation of the immune system in zebrafish, Danio rerio: A gene expression profiling, in situ hybridization and immunological study. Dev. Comp. Immunol. 2004, 28, 9-28. [CrossRef]

39. Novoa, B.; Figueras, A. Zebrafish: Model for the study of inflammation and the innate immune response to infectious diseases. Adv. Exp. Med. Biol. 2012, 946, 253-275.

40. Maggioncalda, E.C.; Story-Roller, E.; Mylius, J.; Illei, P.; Basaraba, R.J.; Lamichhane, G. A mouse model of pulmonary Mycobacteroides abscessus infection. Sci. Rep. 2020, 10, 3690. [CrossRef] [PubMed]

41. Purnapatre, K.P.; Rao, M.; Pandya, M.; Khanna, A.; Chaira, T.; Bambal, R.; Upadhyay, D.J.; Masuda, N. In vitro and in vivo activities of DS86760016, a novel leucy-ltRNA synthetase inhibitor for gram-negative pathogens. Antimicrob. Agents Chemother. 2018, 62, e01987-17. [CrossRef] [PubMed]

42. O'Dwyer, K.; Spivak, A.T.; Ingraham, K.; Min, S.; Holmes, D.J.; Jakielaszek, C.; Rittenhouse, S.; Kwan, A.L.; Livi, G.P.; Sathe, G.; et al. Bacterial resistance to leucyl-tRNA synthetase inhibitor GSK2251052 develops during treatment of complicated urinary tract infections. Antimicrob. Agents Chemother. 2015, 59, 289-298. [CrossRef] [PubMed]

43. Palencia, A.; Li, X.; Bu, W.; Choi, W.; Ding, C.Z.; Easom, E.E.; Feng, L.; Hernandez, V.; Houston, P.; Liu, L.; et al. Discovery of novel oral protein synthesis inhibitors of mycobacterium tuberculosis that target leucyl-tRNA synthetase. Antimicrob. Agents Chemother. 2016, 60, 6271-6280. [CrossRef] [PubMed]

44. Hanh, B.T.B.; Kim, T.H.; Park, J.W.; Lee, D.G.; Kim, J.S.; Du, Y.E.; Yang, C.S.; Oh, D.C.; Jang, J. Etamycin as a novel mycobacterium abscessus inhibitor. Int. J. Mol. Sci. 2020, 21, 6908. [CrossRef] [PubMed]

45. Dubois, V.; Laencina, L.; Bories, A.; Le Moigne, V.; Pawlik, A.; Herrmann, J.L.; Girard-Misguich, F. Identification of virulence markers of mycobacterium abscessus for intracellular replication in phagocytes. J. Vis. Exp. 2018, 139, e57766. [CrossRef] [PubMed] 